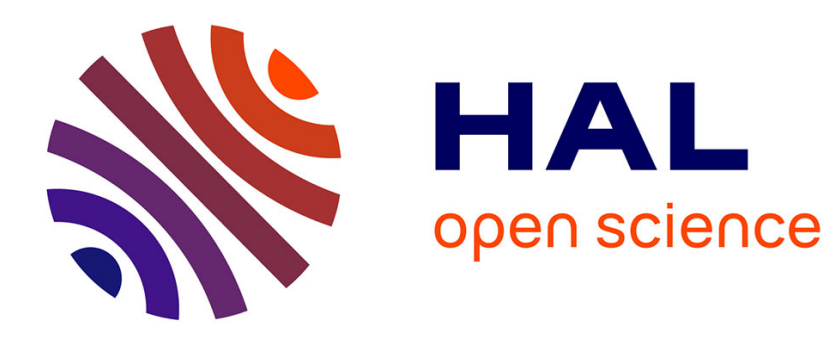

\title{
John Gower ou le multilinguisme en action Aude Mairey
}

\section{To cite this version:}

Aude Mairey. John Gower ou le multilinguisme en action. Médiévales, 2015, 68, pp.57-72. 10.4000/medievales.7533 . halshs-02391972

\section{HAL Id: halshs-02391972 \\ https://shs.hal.science/halshs-02391972}

Submitted on 3 Dec 2019

HAL is a multi-disciplinary open access archive for the deposit and dissemination of scientific research documents, whether they are published or not. The documents may come from teaching and research institutions in France or abroad, or from public or private research centers.
L'archive ouverte pluridisciplinaire HAL, est destinée au dépôt et à la diffusion de documents scientifiques de niveau recherche, publiés ou non, émanant des établissements d'enseignement et de recherche français ou étrangers, des laboratoires publics ou privés. 


\section{John Gower ou le multilinguisme en action}

À la fin du XV $\mathrm{XV}^{\mathrm{e}}$ siècle, George Ashby, membre de la maison des Lancastre et auteur d'un des derniers miroirs au prince poétiques de la période, The Active Policy of a Prince, commence ainsi son œuvre ${ }^{1}$ :

Maittres Gower, Chaucer et Lydgate, premiers poètes de cette nation, [vous avez] pour toujours embelli l'anglais, premiers fondateurs, pour notre réconfort, d'un anglais doux et vivifiant, pour la composition de nouvelles ballades - inédites auparavant - par lesquelles nous pouvons tous acquérir savoir et instruction.

John Gower, né vers 1330 et mort en 1408, issu de la gentry anglaise (c'est-à-dire la petite et moyenne noblesse ${ }^{2}$ ), est considéré par Ashby comme l'un des pères de l'anglais, aux côtés du père absolu, Geoffrey Chaucer, et de John Lydgate, le poète le plus prolifique du XV siècle. De fait, Gower a longtemps été considéré comme un des principaux artisans du «triomphe de l'anglais » à la fin $\mathrm{du} \mathrm{XIV}^{\mathrm{e}}$ et au XV siècle, dans le cadre du récit historiographique - très prégnant jusqu'à ces dernières décennies et porté notamment par John Fisher il y a peu encore ${ }^{3}$.

[p. 58] Or, Gower a écrit son œuvre poétique dans les trois langues alors présentes en Angleterre - le latin d'une part, et les deux langues vernaculaires en circulation d'autre part, le français et l'anglais - et il est le seul poète de la période à l'avoir fait de manière aussi systématique. Même si ses contemporains, à commencer par Chaucer et, un peu plus tard, Thomas Hoccleve et Lydgate, connaissaient, comme lui, les trois langues, leur œuvre poétique est écrite en anglais ${ }^{4}$. Gower, en revanche, a non seulement composé une œuvre trilingue importante, mais il a également inséré des vers et des gloses en latin dans plusieurs de ses œuvres vernaculaires qui revêtent, nous le verrons, des fonctions ambiguës ${ }^{5}$.

\footnotetext{
${ }^{1}$ George Ashby, Active Policy of a Prince, v. 1-7: «Maisters Gower, Chaucer and Lydgate, / Primier poetes of this nacion, / Embelysshing oure englisshe tendure algate, / Firste finders to oure consolacion / Off fresshe, douce englisshe and formacion / Of newe balades, not vsed before, / By whome we all may haue lernyng and lore» (George Ashby's Poems, éd. G. bAteson, Londres, 1899).

2 Pour une biographie de Gower, voir J. Hines, N. Cohen et S. Roffey, «Iohannes Gower, Armiger, Poeta: Records and Memorials of his Life and Death », dans S. Echard éd., A Companion to Gower, Cambridge, 2004, p. 23-42.

${ }^{3}$ J. H. Fisher, The Emergence of Standard English, Lexington, 1996. Pour une mise au point historiographique récente, voir C. Fletcher, «Langue et nation en Angleterre à la fin du Moyen Âge », Revue française d’histoire des idées politiques, 36 (2012), p. 233-252 ; A. Mairey, «Multilinguisme et code-switching en Angleterre à la fin du Moyen Âge. Approche historiographique ", Cabiers électroniques d'bistoire textuelle du LaMOP, 2 (2009) (1re éd. en ligne 2011: http://lamop.univ-paris1.fr/spip.php?rubrique218). Pour une déconstruction systématique de ce paradigme, voir M. C. Davidson, Medievalism, Multilingualism, and Chaucer, New York, 2010.

${ }^{4}$ On trouve toutefois, en français, quelques poèmes peut-être écrits par Chaucer (Chancer and the Poems of "Ch", éd. J. I. Wimsatt, Kalamazoo, 2009). Quant à Hoccleve, qui était un clerc du Sceau privé, il a également laissé un formulaire en français et en latin, mais nous sommes là en dehors du cadre strictement poétique (cf. H. K. Killick, Thomas Hoccleve as Poet and Clerk, PhD, York, 2010).

${ }^{5}$ Les œuvres complètes de Gower ont été éditées au tournant du XIX et du XX ${ }^{\mathrm{e}}$ siècle par George Macaulay (The Complete Works of John Gower, éd. G. C. MACAulAy, 4 vol., Londres, 1899-1902). La Confessio Amantis a été éditée récemment par Russell A. Peck (John Gower, Confessio Amantis, éd. R. A. Peck, Kalamazoo, 3 vol., 2004-2013) ; les ballades en français par Robert Yeager (John Gower, The French Ballades, éd. R. F. Yeager, Kalamazoo, 2011) ; les
} 
Aude Mairey - John Gower ou le multilinguisme en action

Plusieurs questions peuvent donc être posées : quel statut et quelles fonctions Gower accorde-t-il à chacune des langues, dans le cadre de sa réflexion, mais aussi vis-à-vis de son auditoire ? Et surtout, y a-t-il des recoupements entre ses différentes œuvres et, si oui, de quelle nature? Quelques pistes seront ici explorées, à partir d'exemples tirés essentiellement des trois principaux poèmes de Gower, après une rapide présentation des œuvres de ce poète multilingue.

[p. 59] Les cuvres de John Gower

Tableau 1 - Les œuvres de Gower.

\begin{tabular}{|l|l|l|}
\hline \multicolumn{1}{|c|}{ Français } & \multicolumn{1}{|c|}{ Latin } & \multicolumn{1}{c|}{ Anglais } \\
\hline $\begin{array}{l}\text { Mirour de l'Omme } \\
\text { (v. 1376-1379 avec des ajouts } \\
\text { plus tardifs) }\end{array}$ & $\begin{array}{l}\text { Vox Clamantis } \\
\text { (ap. 1381 avec des révisions) }\end{array}$ & $\begin{array}{l}\text { Confessio Amantis* } \\
\text { (1390-1393) }\end{array}$ \\
\hline $\begin{array}{l}\text { Traitié selonc les auctours pour } \\
\text { essampler les amantz. marietz* } \\
\text { (v. 1385-1390) }\end{array}$ & $\begin{array}{l}\text { Cronica Tripertita } \\
\text { (v. 1400) }\end{array}$ & $\begin{array}{l}\text { In Praise of Peace } \\
\text { (v. 1400) }\end{array}$ \\
\hline $\begin{array}{l}\text { Cinkante Balades } \\
\text { (peut-être 1391-1393) }\end{array}$ & Poèmes latins (fin XIVe siècle) & \\
\hline
\end{tabular}

* Gloses latines

Le Mirour de l'Omme $e^{6}$, qui ne subsiste - de manière incomplète - que dans un seul manuscrit conservé à la Bibliothèque universitaire de Cambridge (Additional 3035), doit être daté, selon les spécialistes, de la fin des années $1370^{7}$. Il s'agit d'un long poème évoquant les péchés et les vertus sous forme d'une psychomachie, avant d'aborder, dans une troisième partie, les défauts des différents états de la société. La fin, remaniée vers 1380 ou dans les années suivantes, alors que Gower vivait déjà au prieuré augustin de Sainte-Marie à Southwark, propose un schéma de réconciliation de l'homme avec Dieu, en particulier par la médiation de la Vierge. De l'avis général, c'est un poème extrêmement ambitieux. Robert Yeager, par exemple, le compare (en tout cas quant à ses intentions) au Paradis perdu de Milton ${ }^{8}$.

Les Cinkante Balades, dont le titre fait clairement écho au recueil français intitulé le Livre des Cent Ballades', constituent pour leur part la seule suite poétique en français écrite par un Anglais. Elles ont peut-être été composées entre 1391 et 1393 (mais la date reste discutée), sans doute

poèmes latins courts et le poème In Praise of Peace par Michael Livingstone (John Gower, The Minor Latin Works and In Praise of Peace, éd. M. Livingstone et R. F. Yeager, Kalamazoo, 2005) ; la Cronica Tripertita par David Carlson (John Gower, Poems on Contemporary Events : the Visio Anglie (1381) and Cronica Tripertita (1400), éd. D. R. Carlson, trad. A. G. Rigg, Toronto, 2011).

${ }^{6}$ Ce titre en français se trouve dans le seul manuscrit conservé; mais dans les colophons se trouvant à la fin de plusieurs manuscrits de la Vox Clamantis et de la Confessio Amantis, le poème français est nommé par un titre latin, Speculum Hominis puis Speculum Meditandis. Voir John Gower, Confessio Amantis, éd. R. A. PeckK, vol. 1, en ligne : http://d.lib.rochester. edu/teams/text/peck-gower-confessio-amantis-colophons.

${ }^{7}$ Cf. R. F. Yeager, «Gower's French Audience : The Mirour de l'Omme », The Chaucer Review, 41/2 (2006), p. 111-137.

8 R. F. Yeager, «John Gower's French », dans S. Echard éd., A Companion..., p. 137-152, p. 140.

${ }^{9}$ Les Cent Ballades, poème du XIV siècle composé par Jean le Seneschal avec la collaboration de Philippe d'Artois, comte d'Eu, de Boucicaut le jeune et de Jean de Crésecque, éd. G. Raynaud, Paris, 1905. 
Aude Mairey - John Gower ou le multilinguisme en action

pour Henri de Lancastre, le futur Henri IV, et ne sont conservées, comme le Mirour, que dans un seul manuscrit (London, British Library, Additional [p. 59] 59495) ${ }^{10}$. Quant au Traitié selonc les auctours pour essampler les amantz, marietr, il est plus répandu puisqu'il subsiste dans treize manuscrits (dont un fragment). Il a été composé dans la seconde moitié des années 1380. Malgré sa forme lyrique, il s'agit bien d'une sorte de traité sur les vertus du mariage qui n'est, selon Gower, en rien contradictoire avec l'amour ; on verra qu'il est étroitement associé à la Confessio Amantis.

En latin, Gower a probablement composé la Vox Clamantis après 1381, sans doute à destination du jeune Richard $\mathrm{II}^{11}$. Le livre I est en effet entièrement consacré à une vision cauchemardesque de la révolte de 1381, dans laquelle les révoltés sont assimilés à des animaux, tandis que les livres suivants portent - comme dans la troisième partie du Mirour - sur les divers états de la société, responsables, selon Gower, du mauvais état du pays. Notons toutefois que, pour certains, le livre I aurait été ajouté a posteriori ${ }^{12}$. Quoi qu'il en soit, la Vox subsiste dans dix manuscrits, dont quatre, selon George Macaulay, sont des copies originales. Elle est généralement suivie de la Cronica Tripertita, qui narre la chute de Richard II au profit d'Henri IV et qui, selon David Carlson, son récent éditeur, est fondée sur les Rolls of Parliament, autrement dit sur la version officielle des événements ${ }^{13}$. Outre ces deux textes, Gower a également composé un certain nombre de courts poèmes en latin, que l'on retrouve généralement aux côtés de la $V o x$ Clamantis, mais aussi de la Confessio Amantis.

La Confessio Amantis, composée pour l'essentiel au début des années 1390 en anglais, est le dernier des trois «grands » poèmes écrits par Gower. À notre connaissance, aucun spécialiste n’a expliqué pourquoi la Confessio Amantis portait un titre en latin. Mais cela doit sans doute être mis en relation avec les vers et les gloses en latin qui accompagnent le poème anglais. C'est une œuvre complexe dans laquelle le narrateur se confesse à Genius, le prêtre de Vénus, ce qui est l'occasion pour Gower de présenter une multitude d'exempla couvrant les sujets les plus divers, le tout accompagné de gloses en latin. Le livre VII constitue à lui seul un véritable miroir au prince, inspiré en partie du Trésor de Brunetto Latini. C’est le poème de Gower le plus largement diffusé - et de loin, puisqu'on en connaît actuellement quarante-cinq manuscrits ${ }^{14}$. Peu après la déposition de Richard II, Gower a également écrit In Praise of Peace, destiné à Henri IV.

[p. 61] En volume donc, Gower emploie les trois langues de façon à peu près équilibrée,

\footnotetext{
${ }^{10}$ Cf. R. F. Yeager, «John Gower's Audience : the Ballades », The Chaucer Review, 40/1 (2005), p. 81-105.

${ }^{11}$ Cf. S. Echard, "Gower's 'bokes of Latin' : Language, Politics and Poetry », Studies in the Age of Chaucer, 25 (2003), p. $123-156$.

12 Ibid.

${ }^{13}$ D. R. Carlson, "Gower on Henry IV's Rule: the Endings of the Cronica Tripertita and its Texts », Traditio, 62 (2007), p. 207-236.

${ }^{14}$ Cf. D. Pearsall, «The Manuscripts and Illuminations of Gower's Works », dans S. Echard éd., A Companion..., p. 73-98 ; J. Fredell, « The Gower Manuscripts : Some Inconvenient Truths », Viator, 41/1 (2010), p. 231-250.
} 
Aude Mairey - John Gower ou le multilinguisme en action

avec peut-être un léger avantage pour le latin, surtout si l'on prend en compte la présence de ce dernier dans la Confessio et le Traitié, sous la forme de vers et de gloses. Et si Gower écrit d'abord en français et plus tardivement en anglais, il continue à employer les trois langues tout au long de sa carrière, à l'exception peut-être des toutes dernières années, où il ne compose plus qu'en latin et en anglais. En outre, d'autres indices suggèrent que Gower lui-même était très attaché à la composition dans les trois langues et qu’il y avait beaucoup réfléchi.

\section{Un attachement au multilinguisme}

Un premier indice est constitué par la tombe qu'il s'est fait construire dans le prieuré augustin de Southwark, et qui se trouve aujourd'hui dans la cathédrale rénovée de Southwark. Sur la pierre tombale, Gower est représenté allongé sur ses trois œuvres majeures ${ }^{15}$. Actuellement (le tombeau ayant été restauré), la Vox Clamantis (en latin) se situe au- dessus, le Speculum Meditantis (c'est-à-dire le Mirour de l'Omme, en français) au milieu et la Confessio Amantis (en anglais) en dessous. Mais initialement, c'était le poème français qui se trouvait au-dessus et le poème latin au milieu ${ }^{16}$. Quoi qu'il en soit, cette représentation constitue une éclatante manifestation de l'attachement que Gower portait à l'ensemble de son œuvre, et pas seulement à son œuvre en anglais. Un autre exemple significatif de cet attachement est un court poème en latin. Que Gower en soit l'auteur ou non (cela n'est pas élucidé), il le connaissait sans doute, car ce poème est inséré dans plusieurs manuscrits qu'il a probablement revus lui-même ${ }^{17}$. Gower y est comparé à Virgile et, là encore, l'accent est mis sur son usage des trois langues de l'Angleterre ${ }^{18}$ :

Les mètres de l'Énéide, des Bucoliques et des Géorgiques tissés par Virgile ont depuis longtemps été objets de louange dans les écoles; pour ces trois livres, il est honoré de préférence aux autres poètes et Rome lui accorde ses [p. 61] principales louanges. De même, ô Gower, l'Angleterre est ainsi dotée avec tes trois petits livres où tu accommodes tes écrits aux choses sérieuses. Il a écrit ses poèmes uniquement dans la langue latine, afin qu'ils puissent être appréciés par les notables italiens ; mais il est clair que tu as écrit tes trois poèmes dans trois langues, afin qu'une plus large éducation soit donnée aux hommes: d'abord la langue française, le latin en deuxième et surtout l'anglais, ta langue maternelle, qui complète ton œuvre.

Soulignons que la comparaison est ici plutôt à l'avantage de Gower! Surtout, il est loué pour sa capacité à maîtriser les trois langues de son pays car - et c'est un point important - cela permet,

15 On trouvera une photographie de cette tombe sur la page personnelle de Siân Echard: http://faculty.arts.ubc.ca/sechard/gower.htm.

${ }^{16}$ Cf. J. Hines, N. Cohen et S. Roffey, «Iohannes Gower, Armiger, Poeta... », dans S. Echard éd., A Companion....

${ }^{17}$ S. Echard, « Gower's ‘bokes of Latin’... », p. 126-127.

18 «Eneidos Bucolis », vers 1-12 (The Minor Latin Works...) : «Eneidos, Bucolis, que Georgica metra perhennis / Virgilio laudis serta dedere scolis ; / Hiis tribus ille libris prefertur honore poetis, / Romaque precipuis laudibus instat eis. / Gower, sicque tuis tribus est dotata libellis / Anglia, morigeris quo tua scripta seris. / Illeque Latinis tantum sua metra loquelis / Scripsit, ut Italicis sint recolenda notis ; / Te tua set trinis tria scribere carmina linguis / Constat, ut inde viris sit scola lata magis : / Gallica lingua prius, Latina secunda, set ortus / Lingua tui pocius Anglica complet opus. » 
Aude Mairey - John Gower ou le multilinguisme en action

selon l'auteur, une plus grande diffusion du savoir porté par le poète. La dimension pédagogique de la poésie de Gower, essentielle, est ici soulignée.

Certes, on rencontre ici et là le topos d'humilité cher à de nombreux poètes de la période, surtout dans les compositions de Gower en français. Dans l'envoi de la ballade nº18 du Traitié, par exemple, il s'excuse de sa mauvaise maîtrise de cette langue ${ }^{19}$ :

Al universiteé de tout le monde

Johan Gower ceste Balade envoie ;

Et si jeo n'ai de François la faconde,

Pardonetz moi qe jeo de ceo forsvoie :

Jeo sui Englois, si quier par tiele voie

Estre excusé ; mais quoique nulls en die,

L'amour parfit en dieu se justifie.

On retrouve ce type d'excuse, qui renvoie également au topos de la pratique d'un «mauvais français » par les Anglais, dans le Mirour. Mais, selon Tim Machan, il s'agit surtout d'une stratégie - comme c'est souvent le cas avec les topoï d'humilité, qui permettent plutôt à un auteur de s'affirmer $^{20}$. On notera d'ailleurs que Gower n'emploie ce type d'excuse que pour le français, et non pour le latin.

\section{Des fonctions complémentaires}

Pendant très longtemps, néanmoins, l'historiographie s'est uniquement intéressée à la partie anglaise de l'œuvre de Gower, dans le cadre, notamment, du récit du «triomphe de l'anglais » évoqué en introduction. [p. 62] Depuis quelques années, toutefois, on assiste à une réévaluation de son œuvre en français, principalement par Robert Yeager ${ }^{21}$, et de son œuvre en latin, essentiellement par Siân Echard ${ }^{22}$ : ces deux chercheurs ont donné nombre d'arguments en faveur de l'importance de chacune des langues dans l'œuvre de Gower. Mais il existe aussi une attention nouvelle portée aux relations entre ces trois langues. Soulignons, par exemple, la parution récente d'un recueil intitulé John Gower, Trilingual Poet ${ }^{23}$, ainsi que l'article important de Tim Machan sur l'usage des trois langues par Gower, qui offre un point de vue essentiellement sociolinguistique ${ }^{24}$.

Certes, tous les critiques, y compris R. Yeager et S. Echard, attribuent une fonction plus ou moins précise à chaque langue. C’est le cas notamment pour le latin. Ce dernier se voit conférer

\footnotetext{
${ }^{19}$ Traitié selonc les auctours pour essampler les amantz marietz, Ballade XVIII, vers 22-28 (John Gower, The French Ballades...). 20 T. W. Machan, « Medieval Multilingualism and Gower's Literary Practice», Studies in Philology, 103/1 (2006), p. 125. Voir aussi l'article de C. Lucken dans le présent volume.

21 Voir notamment R. F. Yeager, «The French of Gower... ».

22 Voir par exemple S. Echard, « Gower's ‘bokes of Latin’... ».

${ }^{23}$ E. Dutton et al. éd., John Gower, Trilingual Poet: Language, Translation and Tradition, Cambridge, 2010. Voir l'introduction d'Elisabeth Dutton: "The book is ordered in such a way as to avoid the ghettoization of Gower's French and Latin works, and to encourage comparison between them and the Confessio Amantis, which is generally more familiar to English-speaking Gower scholars » (p. 1).

${ }^{24}$ T. W. Machan, « Medieval Multilingualism... ».
} 
Aude Mairey - John Gower ou le multilinguisme en action

une fonction hautement politique - et pas seulement pour renforcer le conservatisme supposé de Gower. Selon Siân Echard, en effet, les textes latins de Gower, principalement la Vox Clamantis et la Cronica Tripertita, démontrent un sens de la critique, et ce non seulement dans le livre I sur la révolte de 1381, pourtant extrêmement hostile aux révoltés, mais aussi dans les livres suivants qui pointent la responsabilité de tous les états de la société anglaise dans l'éclatement de la révolte ${ }^{25}$. Ces textes révéleraient également une certaine anxiété de la part du poète, au-delà de la dimension «propagandiste » récemment analysée par David Carlson ${ }^{26}$. Toutefois, comme l'a souligné Tim Machan, il n'y a rien de rigide dans la différenciation des langues selon leur fonction ${ }^{27}-$ par exemple, la Confessio possède également une dimension politique importante, encore que celle-ci soit peut-être de nature un peu plus générale, comme en témoigne en particulier le livre VII qui constitue, on l'a dit, un véritable miroir au prince.

[p. 64] Une fonction spécifique attribuée à une langue peut également être en partie brouillée. Le latin possède aussi, évidemment, une fonction d'autorité, particulièrement perceptible dans les vers et les gloses en latin de la Confessio, mais aussi du Traitié. Leur fonction de légitimation semble ici évidente. Toutefois, les choses ne sont en réalité pas si tranchées et la véritable nature de ces insertions a fait couler beaucoup d'encre, en particulier parce que, comme l'a montré Siân Echard, les gloses sont parfois en contradiction avec le texte anglais : « Le latin de Gower problématise la question de l'autorité dans la Confessio, en présentant au lecteur plusieurs voix d'autorité, en latin et en langue vernaculaire, dont aucune ne semble capable de dominer le $\operatorname{texte}^{28}$. $\gg$ Le cas du latin suggère donc que, même si les trois langues peuvent avoir des fonctions différenciées, les frontières entre elles sont floues et les ponts nombreux.

\section{Une interpénétration profonde}

Comme l'avait déjà remarqué en son temps George Macaulay, il existe, si ce n'est une interpénétration profonde entre les œuvres de Gower, du moins une grande proximité. À tel point que Tim Machan a pu se demander si Gower n'avait pas écrit trois fois la même œuvre ${ }^{29}$. Sa

\footnotetext{
${ }^{25}$ S. Echard, « Gower's 'bokes of Latin'... ».

${ }^{26}$ D. R. Carlson, John Gower: Poetry and Propaganda in Fourteenth-Century England, Cambridge, 2012. L'emploi de ce terme de propagande reste toutefois problématique pour le Moyen Âge : cf. A. Mairey, «Les langages politiques au Moyen Âge. Introduction », Médiévales, 57 (2009), p. 5-14.

${ }^{27}$ T. W. Machan, « Medieval Multilingualism... », p. 111-112 : «In very general terms [...], Latin may well have been the language of authority, French of the court and law, and English of daily experience. Within these general terms, however, were opportunities for the exploitation of the subtleties and nuances of language contact in medieval England. » Sur ce point, mais pour une interprétation un peu différente, voir M. C. Davidson, Medievalism..., p. 4674.

${ }^{28}$ S. Echard, «With Carmen's Help : Latin Authorities in the Confessio Amantis», Studies in Philology 95/1 (1998), p. 140: "Gower's Latin problematizes the question of authority in the Confessio by presenting a reader with several competing authoritative voices, Latin and vernacular, none of which seems capable of taming the text » (p. 7).

${ }^{29}$ T. W. Machan, « Medieval Multilingualism... », p. 1-2.
} 
Aude Mairey - John Gower ou le multilinguisme en action

réponse est bien sûr négative. Toutefois, en un sens, il semble possible de parler non seulement de translatio "externe", puisque la Vox Clamantis, par exemple, est en grande partie une compilation d'auteurs anciens (à commencer par Ovide), mais aussi de translatio «interne », dans la mesure où l'on observe, pour certains passages, mais aussi pour des structures générales, des connexions extrêmement fortes entre les différents poèmes.

Il faut souligner, en premier lieu, qu'il existe de nombreuses similarités sur le plan formel entre le Mirour et la Confessio, mais aussi, dans une moindre mesure, entre ces deux derniers et la Vox Clamantis. Nous ne nous y attarderons pas car Tim Machan, après Macaulay, en a donné de nombreux exemples. Ce dernier avait déjà insisté sur les similitudes entre les poèmes français et anglais $^{30}$ :

[p. 65] Les deux sont écrits dans le même vers octosyllabique, avec la même régularité métrique, plutôt monotone $[\ldots]$; dans la structure de la phrase, on trouve certaines caractéristiques définies qui surviennent de la même manière dans les œuvres françaises et anglaises.

Certains parallèles sont également très frappants au niveau lexical, surtout entre les deux langages vernaculaires - ce qui n'est toutefois guère surprenant au vu de l'importance du transfert lexical du français vers l'anglais à cette époque ${ }^{31}$.

Sur le plan du contenu, il est évidemment impossible de tout passer en revue, car les trois poèmes principaux sont très longs. Nous ne prendrons qu'un exemple, celui des états de la société, dont le traitement dans les trois poèmes est résumé dans le tableau suivant:

Tableau 2 - Concordances thématiques des œuvres de Gower concernant

les états de la société

\begin{tabular}{|l|l|l|l|}
\hline & \multicolumn{1}{|c|}{ Mirour de l'Omme } & \multicolumn{1}{c|}{ Vox Clamantis } & \multicolumn{1}{c|}{ Confessio Amantis } \\
\hline $\begin{array}{l}\text { Considérations } \\
\text { générales }\end{array}$ & $\begin{array}{l}\text { VIII, 26509-26604 } \\
\text { (« He, Siecle... })\end{array}$ & $\begin{array}{l}\text { II, 1-50; VII, 1159- } \\
\text { 1444 }\end{array}$ & $\begin{array}{l}\text { Prol. 119-150; 520- } \\
534\end{array}$ \\
\hline Fortune & V, 22081-22164 & II, 51-216 & Prol. 125-150 \\
\hline Guerre et paix & VI, 24097-24168 & & $\begin{array}{l}\text { Prol. 122-192 (+ livre } \\
\text { III) }\end{array}$ \\
\hline Église & III, 18421-21780 & III-IV & Prol. 193-418 \\
\hline Pape et prélats & III, 18421 sq. & III, 79 sq. & \\
\hline La guerre] & III, 18649-18708 & III, 329-956 & Prol. 240-283 \\
\hline $\begin{array}{l}\text { Prédication et } \\
\text { exemplarité }\end{array}$ & $\begin{array}{l}\text { III, 19069-19344 (à } \\
\text { propos des évêques) }\end{array}$ & III, 1005-1192 & Prol. 452-498 \\
\hline Curés & III, 20209-20496 & III, 1313-1554 & \\
\hline $\begin{array}{l}\text { Prêtres non- } \\
\text { bénéficiaires }\end{array}$ & III, 20497-20640 & III, 1555-1672 & \\
\hline Temps passé/présent & III, 20641-20784 & III, 1833-2048 & Prol. 193-239 \\
\hline Les jeunes & III, 20785-20830 & III, 2049-2142 & \\
\hline
\end{tabular}

${ }^{30}$ The Complete Works..., éd. G. C. Macaualy, vol. 1, p. xl : «Both are in the same octosyllabic line, with the same rather monotonous regularity of metre $[\ldots]$; in the structure of the sentence, there are certain definite characteristics which produce themselves equally in the French and English works. »

${ }^{31}$ Cf. A. C. Baugh et T. Cable, A History of English Language, fifth edition, Londres, 2002, p. 163 sq. 
Aude Mairey - John Gower ou le multilinguisme en action

\begin{tabular}{|l|l|l|l|}
\hline Moines (et chanoines) & IV, 20831-21180 & IV, 1-676 & \\
\hline Frères & IV, 21181-21780 & IV, 677-1232 & \\
\hline $\begin{array}{l}\text { [p. 66] État et } \\
\text { aristocratie }\end{array}$ & V, 21781-25176 & V-VI & $\begin{array}{l}\text { Prol. 93-192; épil. } \\
3054-3105\end{array}$ \\
\hline Nabuchodonosor & $\begin{array}{l}\text { V, [...] 21979-21996; } \\
22765-\end{array}$ & VII, 1-216 & $\begin{array}{l}\text { Prol. 585-820; V, } \\
7017 \text { sq. }\end{array}$ \\
\hline Peuples d'Israël & V, 22009-22056 & & Prol. 535-584 \\
\hline Le roi & $\begin{array}{l}\text { V, 22225-23208 } \\
\text { (David, 22920-22980) }\end{array}$ & VI, 469-1122 & $\begin{array}{l}\text { Prol. 173-192 ; épil. } \\
\text { 3054-3105; VII. }\end{array}$ \\
\hline Barons et chevaliers & VI, 23209-24180 & V, 1-556 & $\begin{array}{l}\text { Certains éléments dans } \\
\text { les livres III et IV. }\end{array}$ \\
\hline $\begin{array}{l}\text { Hommes de loi et } \\
\text { officiers }\end{array}$ & VII, 24181-25176 & VI, 1-468 & \\
\hline Communes & VIII, 25177-26508 & V & $\begin{array}{l}\text { Prol. 499-584 } \\
\text { (indistinctes) }\end{array}$ \\
\hline Marchands & $\begin{array}{l}\text { VIII, 25177-25500 (la } \\
\text { laine, 25369-25428) }\end{array}$ & V, 655-786 & \\
\hline Artisans & VIII, 25501-25785 & V, 787-834 & \\
\hline Marchands et artisans & VIII, 25786-25980 & V, 835-1016 & Prol. 111-118 \\
\hline «Vitaillers'» & VIII, 25981-26424 & & \\
\hline Paysans & VIII, 26425-26508 & V, 557-654 & \\
\hline
\end{tabular}

Gower évoque les différents états de la société de manière très détaillée dans les poèmes français et latin, et de manière moins développée dans le prologue de la Confessio ${ }^{32}$. Même si le traitement est plus succinct dans le prologue du poème anglais, on retrouve toutefois des parallèles évocateurs : les considérations sur la Fortune par exemple, ou encore l'histoire de Nabuchodonosor, chère au cœur du poète - qui, par ailleurs, est souvent employée par les écrivains de la période ${ }^{33}$. Mais d'autres parallèles existent, en dehors d'exempla suremployés, par exemple la question de l'implication de certains membres du clergé dans la justification de la guerre, voire de leur participation à des activités militaires. Cela provoque un vif mécontentement [p. 67] chez Gower et les clercs incriminés sont fortement critiqués dans les trois poèmes - et ce alors même que les considérations plus générales sur la guerre et la paix n’apparaissent pas dans la Vox Clamantis.

En ce qui concerne la différenciation des trois ordres de la société, elle est très précise dans le poème latin et plus encore dans le Mirour de l'Omme. Gower ne se contente pas de broder sur le schéma général des trois ordres, même s’il l'évoque (notamment dans le Mirour) et qu'il structure globalement son propos à partir de ce schéma. Il est, au contraire, très minutieux. Il commence par passer en revue tous les membres du clergé séculier et régulier, y compris les étudiants, les clercs sans bénéfices et les chanoines. Il insère de longs développements sur les hommes de loi,

\footnotetext{
32 Mais on retrouve des considérations plus étoffées dans le développement du poème : c'est le cas, par exemple, des barons et des chevaliers qui apparaissent notamment dans les livres III et IV.

${ }^{33}$ Qu'ils soient poètes ou non : cf. A. Mairey, " "Pour la charité et le commun profit" : Bible, hérésie et politique en Angleterre ", Cabiers électroniques d'histoire textuelle du LaMOP, 3, 2010 (1 re éd. en ligne 2011 : http://lamop.univparis1.fr/spip.php?rubrique223).
} 
Aude Mairey - John Gower ou le multilinguisme en action

qu'il connaît vraisemblablement très bien - il a peut-être reçu une formation juridique ${ }^{34}$. Il établit de nettes distinctions entre les magnats d'une part et la gentry d'autre part, même s'il reconnait qu'ils partagent un certain nombre de défauts. Il en est de même pour les différents types de marchands et d'artisans. Gower nous livre par exemple, dans le Mirour, un long passage sur les taverniers et leurs mauvaises pratiques. Les développements sur la laine, nerf économique de l'Angleterre, en particulier dans le Mirour, suggèrent d'ailleurs que Gower est très au fait des conditions économiques contemporaines.

Cette attention portée à la complexité de la société anglaise de son époque, même si elle est empreinte de nombreux stéréotypes sur les défauts supposés des uns et des autres ${ }^{35}$, est dans l'air du temps. Comme nous l'avons montré ailleurs, ses contemporains, notamment William Langland et Geoffrey Chaucer, sont également - et peut-être plus encore - sensibles à cette complexité dont le schéma des trois ordres ne rend plus suffisamment compte. Mais cette attention n'est pas uniquement le fait des écrivains : on la retrouve aussi, par exemple, dans la loi somptuaire de 1363 qui tente de gommer en partie cette complexités. Gower, pour sa part, justifie chaque fois l'existence même de ces groupes, comme en témoigne l'exemple des marchands dans le Mirour $^{37}$ :

[p. 68] Si une terre avoir porroit

Tous biens ensemble, lors serroit

Trop orguillouse, et pour cela

Dieu establist, et au bon droit,

Qe l'une terre en son endroit

Del autry bien busoignera :

Sur quoy marchant dieus ordina,

Qui ce q'en l'une ne serra

En l'autre terre querre doit:

Pour ce qui bien se gardera,

Et loyalment marchandera,

De dieu et homme il est benoit.

Et de fait, en tout cas dans le Mirour qui développe le plus ces questions, Gower commence toujours par justifier l'existence d'une catégorie (en en rappelant les devoirs), avant de s'attaquer à ses défauts. Et il est particulièrement sensible à la nécessaire unité des différentes parties de la société, tout en déplorant constamment ses divisions. De toute façon, il est clair que Gower, en y insistant dans chacun de ses grands poèmes, entend sensibiliser l'ensemble de ses lecteurs à

\footnotetext{
34 Cf. D. Gray, «Gower, John (d. 1408)», Oxford Dictionary of National Biography, Oxford, 2004 [http://www.oxforddnb.com.janus.biu.sorbonne.fr/view/article/11176, consulté le 22 avril 2014].

${ }^{35}$ Dans la Vox Clamantis, par exemple, les dénonciations du clergé sont pour l'essentiel reprises du De vita monachorum d'Alexandre Nequam ou du Speculum stultorum de Nigel de Longchamp. Cf. The Complete Works..., éd. G. C. Macaulay, vol. 4, p. xxxii-xxxiii.

${ }^{36}$ Cf. A. Mairey et F. Collard, «In the Mirror of Mutual Representation : Political Society as Seen by Its Members », à paraitre dans C. Fletcher, J.-P. Genet et J. Watts éd., Government and Political Life in England and France, c. 1300-c. 1500, Cambridge.

${ }^{37}$ Mirour de l'omme, v. 25189-25200.
} 
l’indispensable réforme morale de chacun de ces groupes.

Cela ne signifie pas pour autant que les différences sont absentes et l'on peut assez aisément distinguer les poèmes français et latin d'une part, tout entiers axés sur ce que Gower appelle la «sagesse », et la Confessio Amantis d'autre part, qui est à la fois, selon son auteur, un livre de sagesse et un livre de divertissement ${ }^{38}$ :

De ceux qui ont écrit avant nous, les livres restent, et nous sommes donc instruits par ce qui a été écrit auparavant; c'est pourquoi il est bon que nous aussi, durant notre temps ici-bas, renouvelions certaine matière illustrée par ces sages anciens, afin qu'elle puisse, de cette manière, être laissée à l'oreille du monde dans les temps à venir après celui-ci, lorsque nous serons morts, et en un autre lieu.

[p. 69] Mais l'on dit, et cela est vrai, que celui qui écrit sur la seule sagesse lasse souvent les esprits de ceux qui liront cela la journée durant; c'est pourquoi, si vous en êtes d'accord, j'emprunterai une voie médiane et j'écrirai un livre entre les deux, en partie pour le plaisir, en partie pour l'enseignement, afin que certains puissent apprécier ce que j'écris d'un côté ou de l'autre, plus ou moins.

Il faut également distinguer entre la Vox Clamantis d'une part, qui est le poème le plus ouvertement politique dans la mesure où il ne traite, à la suite du livre I sur la révolte de 1381, que des états de la société, des groupes sociaux; et le Mirour et la Confessio d'autre part, qui mettent considérablement l'accent sur la réforme de l'individu, en particulier par l'intermédiaire de longs développements sur les péchés et les vertus.

\section{L'anditoire et le public de Gower face au multilinguisme}

Sans doute, comme Robert Yeager, faut-il voir dans ces distinctions un lien avec des publics et des auditoires différenciés selon la langue employée - nous reprenons ici la distinction établie par Anne Middleton, selon laquelle le public est «le lectorat imaginé et posé par l'écrivain » et l'auditoire «le lectorat attesté par des preuves»" Notons toutefois que, dans tous les cas, le prince est au pinacle de ces auditoires, même s'il n'est pas l'unique destinataire des œuvres de Gower. Le Mirour semble avoir été destiné d'abord à Édouard III et à sa cour, et Robert Yeager a montré qu'il avait des affinités avec le Livre des Seintes Medecines d'Henri de Lancastre ${ }^{40}$. Le poème aurait toutefois été réorienté par Gower, dans les années 1380, en direction des Augustins du prieuré de Sainte-Marie à Southwark ${ }^{41}$ - ce qui suggère non seulement que certains membres du clergé ne répondaient pas uniquement au latin, mais qu'ils étaient également susceptibles de

\footnotetext{
38 John Gower, Confessio Amantis, v. 1-21: «Of hem that writen ous tofore / The bokes duelle, and we therfore / Ben tawht of that was write tho : / Forthi good is that we also / In oure tyme among ous hiere / Do wryte of newe som matiere, / Essampled of these olde wyse, / So that it myhte in such a wyse, / Whan we ben dede and elleswhere, / Beleve to the worldes eere / In tyme comende after this. / Bot for men sein, and soth it is, / That who that al of wisdom writ / It dulleth ofte a mannes wit / To him that schal it aldai rede, / For thilke cause, if that ye rede, / I wolde go the middel weie / And wryte a bok betwen the tweie, / Somwhat of lust, somewhat of lore, / That of the lasse or of the more / Som man mai lyke of that I wryte. » Sur cette question, voir A. Mairey, «La translatio poétique en Angleterre à la fin du Moyen Âge », à paraitre dans A. Thompson et J.-P. Genet éd., Traductions et culture dans les Iles Britanniques : une approche historique.

39 A. Middleton, «The Audience and Public of Piers Plowman », dans Middle English Alliterative Poetry and its Background, éd. D. Lawton, Woodbridge, 1982, p. 101-123.

${ }^{40}$ Henri de Lancastre, Le Livre des Seynts Medicines, éd. E. J. Arnould, Oxford, 1940.

${ }^{41}$ R. F. Yeager, «Gower's French Audience... ».
} 
Aude Mairey - John Gower ou le multilinguisme en action

s'intéresser aux idées d'un laïc singulier, qui fut en résidence dans ce prieuré pendant près des trois dernières décennies de sa vie. Notons que ce ne sont pas les seuls membres du clergé régulier anglais à s'intéresser à la littérature vernaculaire; les Chartreux, par exemple, ont également été actifs en matière de diffusion d'une littérature de dévotion en anglais ${ }^{42}$. La $V o x$ Clamantis est, pour sa part, clairement destinée au jeune Richard II, mais sa diffusion - dix manuscrits conservés, on l'a vu - suggère un lectorat un peu [p. 70] plus large, probablement clérical pour l'essentiel. La Confessio Amantis, enfin, a d'abord été dédicacée à Richard II, puis à Henri de Lancastre. Mais pour le poème anglais, l'auditoire induit aussi bien que réel est beaucoup plus large ${ }^{43}$. En l'occurrence, seul l'usage de la langue maternelle peut permettre de s'adresser à tous les états de la société anglaise mentionnés par Gower, tandis que le latin, mais aussi le français, possèdent une dimension plus «internationale». Et c'est sans doute une des raisons pour lesquelles la Confessio a davantage été diffusée que les autres textes, et ce de manière tout à fait consciente, si l'on en croit la majorité des spécialistes de l'histoire de ses manuscrits ${ }^{44}$.

Toutefois, comme l'ont déjà souligné plusieurs critiques, ce n’est pas seulement Gower qui exploite consciencieusement son multilinguisme, mais aussi ses copistes ou ses lecteurs ${ }^{45}$. Plusieurs manuscrits de ses œuvres sont en effet eux-mêmes multilingues. Le Traitié selonc les auctours pour essampler les amantz marietz, par exemple, accompagne la Confessio dans huit manuscrits - soit près des trois quarts des manuscrits subsistants du poème en français. Dans l'esprit même de Gower, d'ailleurs, il semble y avoir un lien assez précis entre ces deux ouvrages, comme le suggère le titre donné au Traitié dans le manuscrit Fairfax 3 de la Bibliothèque Bodléienne d'Oxford ${ }^{46}$ :

Puisqu'il ad dit ci devant en Englois par voie d'essample la sotie de cellui qui par amours aime par especial, dirra ore apres en François a tout le monde en general un traitié selonc les auctours pour essampler les amantz marietz, au fin q'ils la foi de lour seintes espousailes pourront par fine loialté guarder, et al honour de dieu salvement tenir.

Mais certains de ces manuscrits comprennent aussi au moins un poème en latin, le Carmen super multiplici viciorum pestilencia. C'est le cas par exemple des manuscrits Fairfax 3, Harley 3869 (Londres, British Library), Bodley 294 (Oxford, Bodleian Library), Cambridge, Trinity College, R.3.2, et Genève, Fondation Bodmer, 178. Or, on sait qu'au moins deux de ces manuscrits ont appartenu à la noblesse ou à la gentry : le Bodley 294 est signé par Humphrey de Gloucester, un des fils d'Henri IV, célèbre bibliophile et mécène, et le Trinity College R.3.2 porte le nom

\footnotetext{
${ }^{42}$ Cf. M. Sargent, «The Transmission by the English Carthusians of Some Late Medieval Spiritual Writings », Journal of Ecclesiastical History, 27 (1976), p. 225-240.

${ }^{43}$ Voir notamment G. Shuffleton, «Romance, Popular Style and the Confessio Amantis: Conflict or Evasion? », dans

E. Dutton et al. éd., John Gower, Trilingual Poet..., p. 74-84.

${ }^{44}$ Voir notamment J. Fredell, «The Gower Manuscripts... ».

${ }^{45}$ Sur ce point, voir T. W. Machan, « Medieval Multilingualism... ».

46 The Complete Works..., éd. G. C. Macaulay, vol. 1, p. 379.
} 
Aude Mairey - John Gower ou le multilinguisme en action

d'Elizabeth Vernon, probablement issue de la gentry. Certes, le contenu latin est minimal par rapport aux deux vernaculaires, mais il est bien présent.

[p. 71] Notons cependant que des trois grands poèmes, seule la Confessio est accompagnée de textes d'autres langues, en tout cas dans un premier temps puisque les manuscrits les plus tardifs sont beaucoup plus homogènes sur le plan linguistique. Cela confirme tout de même que certains lecteurs de Gower, au moins, ne sont pas monolingues et apprécient la diversité de ses compositions. Soulignons néanmoins qu'au début du XV siècle, un certain Quixley, un clerc du nord de l'Angleterre, ressent le besoin de traduire le Traitié en anglais ${ }^{47}$.

Le cas de John Gower est certes exceptionnel, dans la mesure où il est le seul poète de sa génération à avoir écrit de manière systématique - et ce, on l'a vu, de manière extrêmement réfléchie - dans les trois langues. Même si la Confessio a connu une diffusion bien plus importante que le Mirour ou la Vox, ce qui explique sans doute aussi pourquoi elle a longtemps fait l'objet de toutes les attentions, l'œuvre anglaise ne peut, finalement, être comprise que dans son environnement multilingue - et pas seulement au regard des vers et des gloses latines qui l'accompagnent, même si ces dernières constituent à elles seules un élément très original dans la poésie anglaise contemporaine. Quelle que soit la langue dans laquelle il écrit, Gower égrène ses préoccupations concernant l'état de la société anglaise et les possibilités de salut de ses compatriotes. Ses successeurs ont privilégié entièrement l'anglais. Mais à la fin du XIV siècle, le médium linguistique est, en Angleterre, encore multiple, et Gower n'a eu de cesse d'être le représentant de cette complexité, en la mettant au service de sa participation au dialogue politique de son époque.

\footnotetext{
${ }^{47}$ Cette traduction a été éditée par R. Yeager dans le volume consacré aux ballades (John Gower, French Balades...). Pour une évaluation de l'identification de Quixley - la plus probable à ce jour étant sans doute celle impliquant Robert de Quixley dans le Yorkshire, supérieur du prieuré augustin de Nostell et titulaire d'une prébende à Bramham de 1393 jusqu'à sa mort en 1427, voir R. F. Yeager, «John Gower's French and His Readers », dans E. Dutton et al. éd., John Gower, Trilingual Poet..., p. 304-314 (p. 307-309).
} 